Supporting information

\title{
Multifunctional Noble Metal Phosphide Electrocatalysts for the Organic Molecule Electro-Oxidation
}

Zhipeng Yu, ${ }^{\dagger},+$ Xian-Kui Wei, ${ }^{\|}$Junyuan Xu, ${ }^{\dagger}$ Yue Li, ${ }^{\dagger}$ Ana Araujo, ${ }^{\dagger},+$ Joaquim L. Faria, ${ }^{\dagger}$ Rafal E. Dunin-Borkowski, ${ }^{\|}$and Lifeng Liu ${ }^{\dagger *}$

${ }^{\dagger}$ International Iberian Nanotechnology Laboratory (INL), Avenida Mestre Jose Veiga, 4715-330 Braga, Portugal

Laboratory of Separation and Reaction Engineering - Laboratory of Catalysis and Materials (LSRE-LCM), Faculdade de Engenharia, Universidade do Porto, Rua Dr. Roberto Frias s/n 4200465 Porto, Portugal

"Ernst Ruska-Centre for Microscopy and Spectroscopy with Electrons, Forschungszentrum Jülich GmbH, Jülich 52428, Germany

AUTHOR INFORMATION

*Corresponding Author

lifeng.liu@inl.int (L. Liu) 


\section{Supporting Figures}
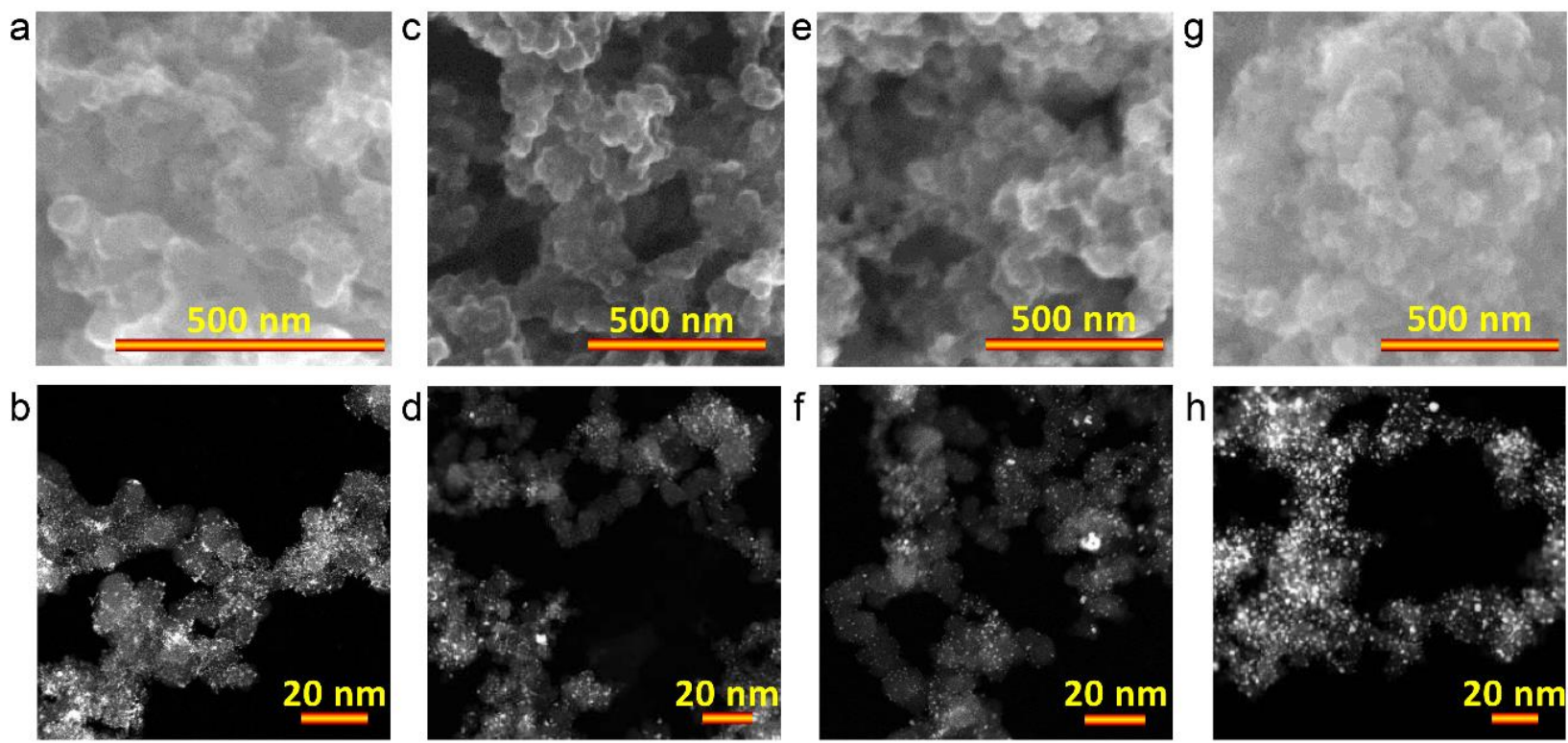

Figure S1. SEM and low-magnification HAADF-STEM images of (a-b) commercial Pd/C, (c-d) $\mathrm{PdP}_{2} / \mathrm{C}$, (e-f) $\mathrm{PdP}_{2}-\mathrm{Pd} / \mathrm{C}$ and $(\mathrm{g}-\mathrm{h}) \mathrm{Pd}_{5} \mathrm{P}_{2}-\mathrm{Pd} / \mathrm{C}$ catalysts derived from the simple one-step phosphorization. 

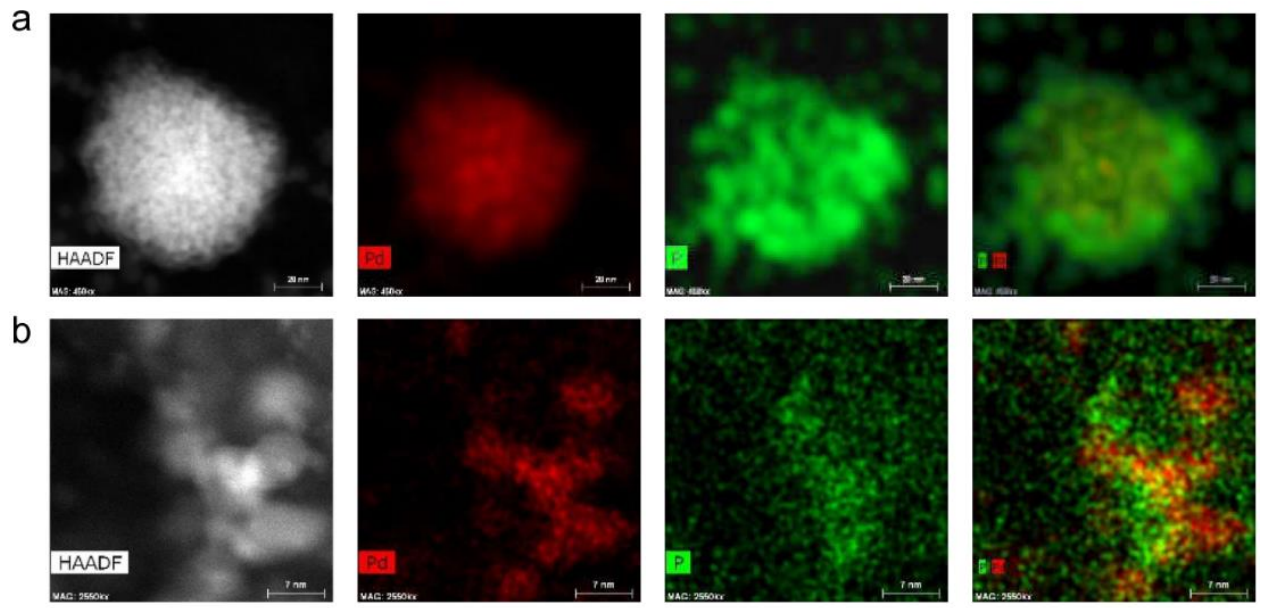

C
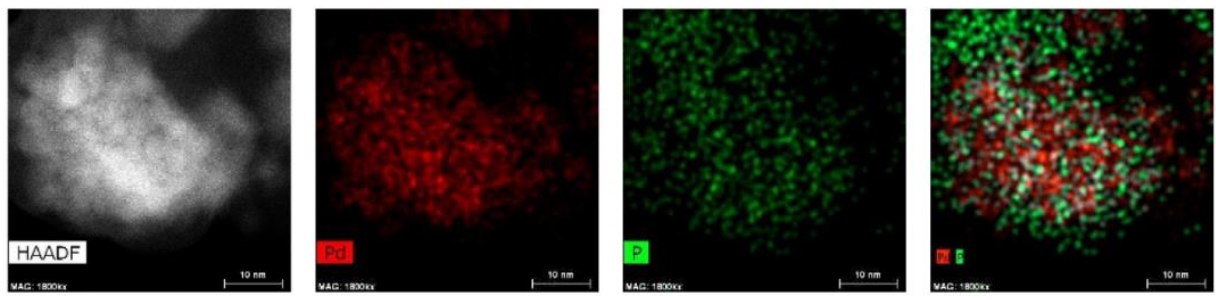

Figure S2. HAADF-STEM image of (a) $\mathrm{PdP}_{2} / \mathrm{C}$, (b) $\mathrm{PdP}_{2}-\mathrm{Pd} / \mathrm{C}$ and (c) $\mathrm{Pd}_{5} \mathrm{P}_{2}-\mathrm{Pd} / \mathrm{C}$ and the corresponding elemental maps of $\mathrm{Pd}, \mathrm{P}$ and their overlay. 


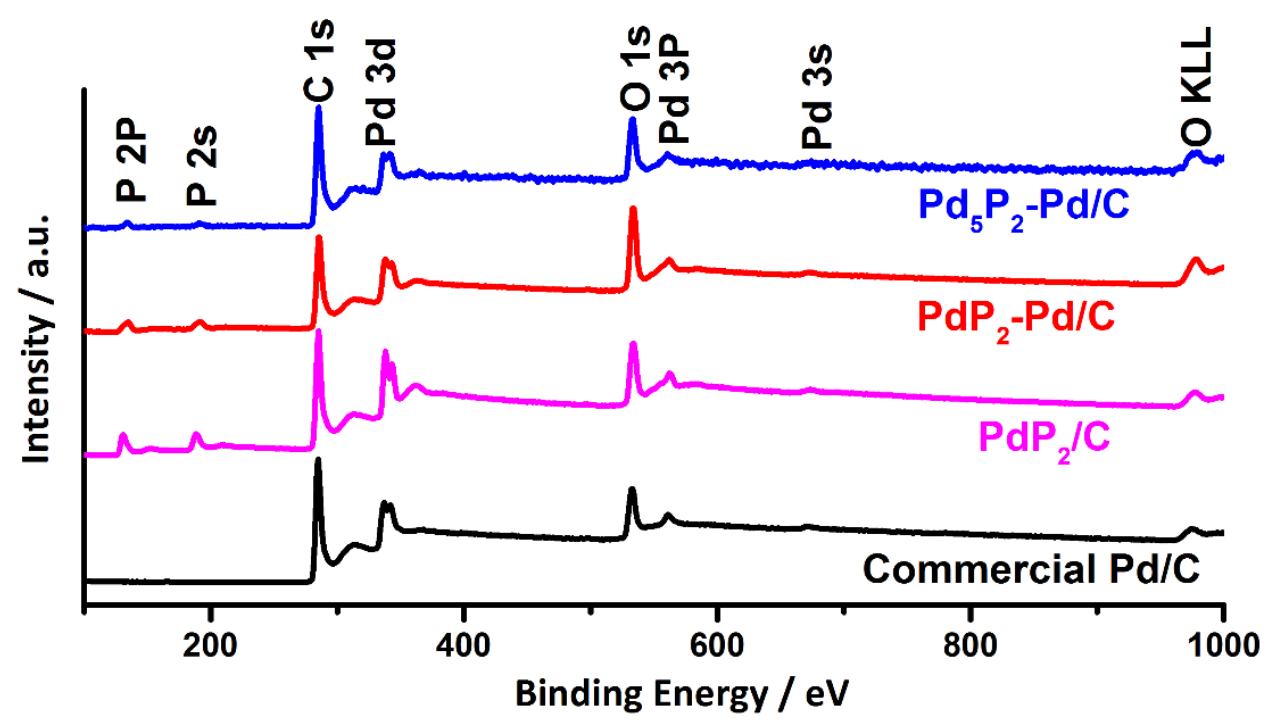

Figure S3. XPS survey spectra of commercial $\mathrm{Pd} / \mathrm{C}, \mathrm{PdP}_{2} / \mathrm{C}, \mathrm{PdP}_{2}-\mathrm{Pd} / \mathrm{C}$ and $\mathrm{Pd}_{5} \mathrm{P}_{2}-\mathrm{Pd} / \mathrm{C}$ catalysts. 

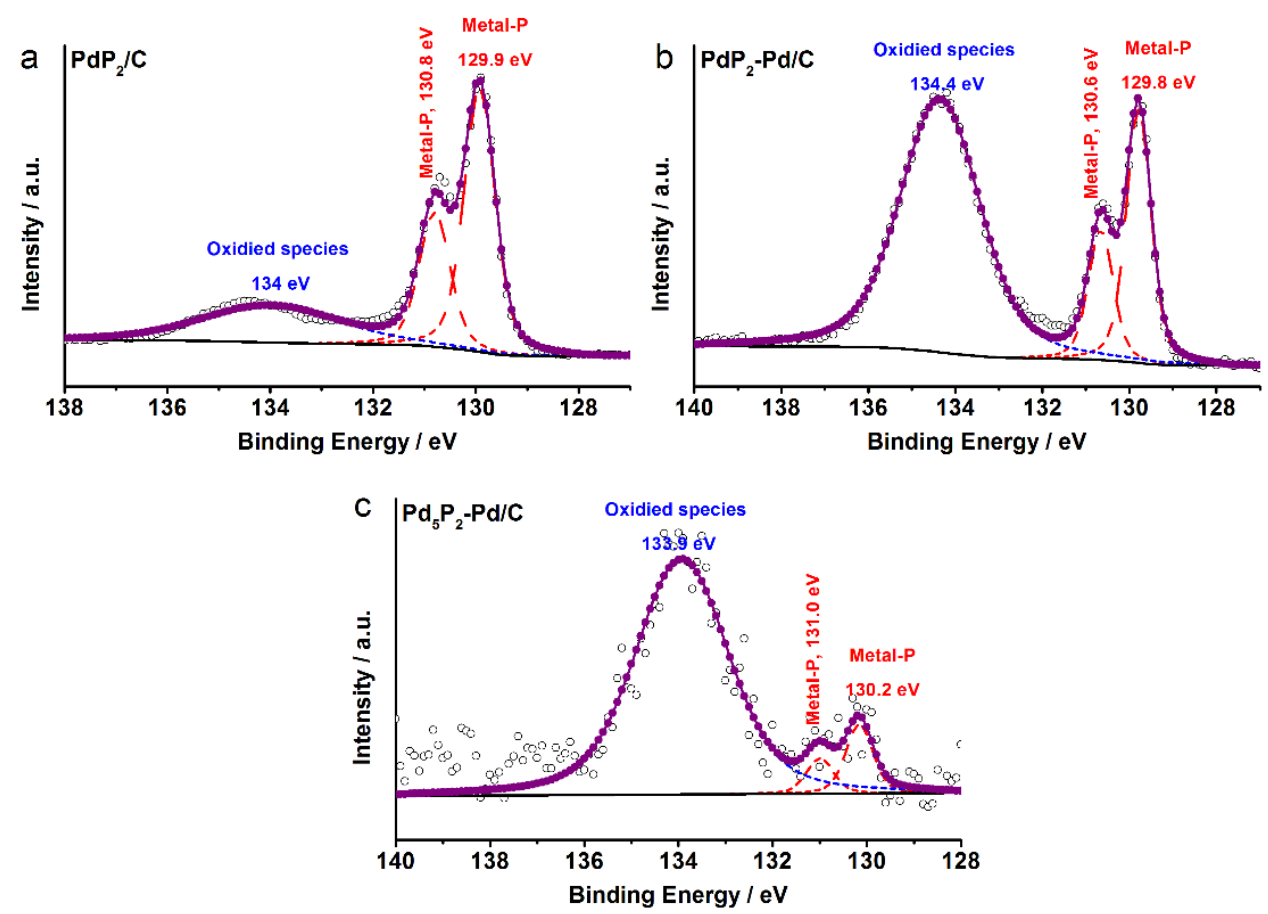

Figure S4. High-resolution $\mathrm{P} 2 \mathrm{p}$ XPS spectra of (a) $\mathrm{PdP}_{2} / \mathrm{C}$, (b) $\mathrm{PdP}_{2}-\mathrm{Pd} / \mathrm{C}$ and (c) $\mathrm{Pd}_{5} \mathrm{P}_{2}-\mathrm{Pd} / \mathrm{C}$ catalysts. 

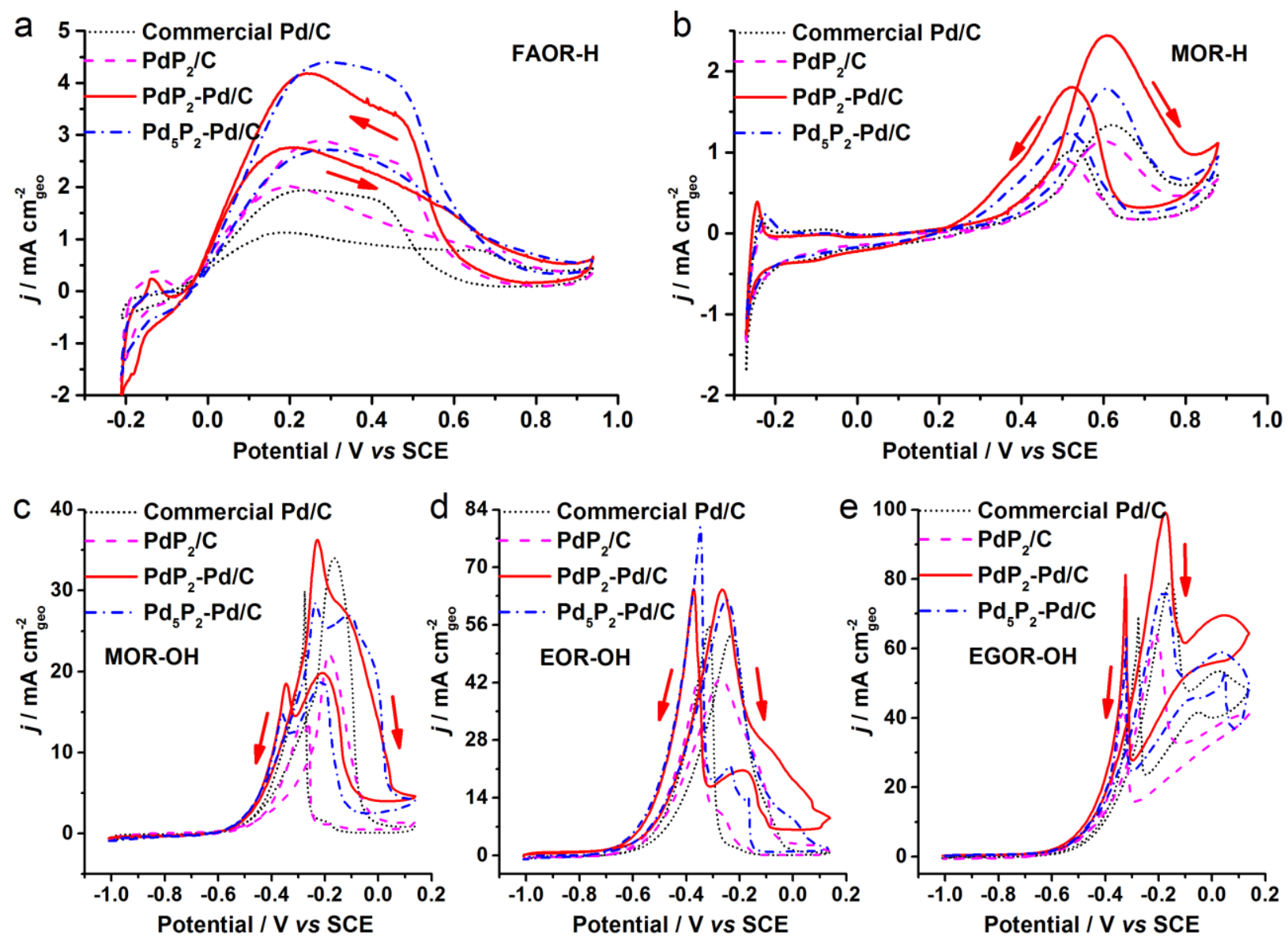

Figure S5. Electrocatalytic performance of commercial $\mathrm{Pd} / \mathrm{C}, \mathrm{PdP}_{2} / \mathrm{C}, \mathrm{PdP}_{2}-\mathrm{Pd} / \mathrm{C}$ and $\mathrm{Pd}_{5} \mathrm{P}_{2}-\mathrm{Pd} / \mathrm{C}$ catalysts toward different OMEO reactions. (a) FAOR-H recorded in $0.5 \mathrm{M} \mathrm{HCOOH}+1.0 \mathrm{M}$ $\mathrm{HClO}_{4}$. (b) MOR-H recorded in $0.5 \mathrm{M} \mathrm{CH}_{3} \mathrm{OH}+0.1 \mathrm{M} \mathrm{HClO}_{4}$. (c) MOR-OH recorded in $1.0 \mathrm{M}$ $\mathrm{CH}_{3} \mathrm{OH}+1.0 \mathrm{M} \mathrm{KOH}$. (d) EOR-OH recorded in $1.0 \mathrm{M} \mathrm{CH}_{3} \mathrm{CH}_{2} \mathrm{OH}+1.0 \mathrm{M} \mathrm{KOH}$. (e) EGOR$\mathrm{OH}$ recorded in 1.0 M EG + 1.0 M KOH. All CV curves were recorded at $20 \mathrm{mV} \mathrm{s}^{-1}$. 

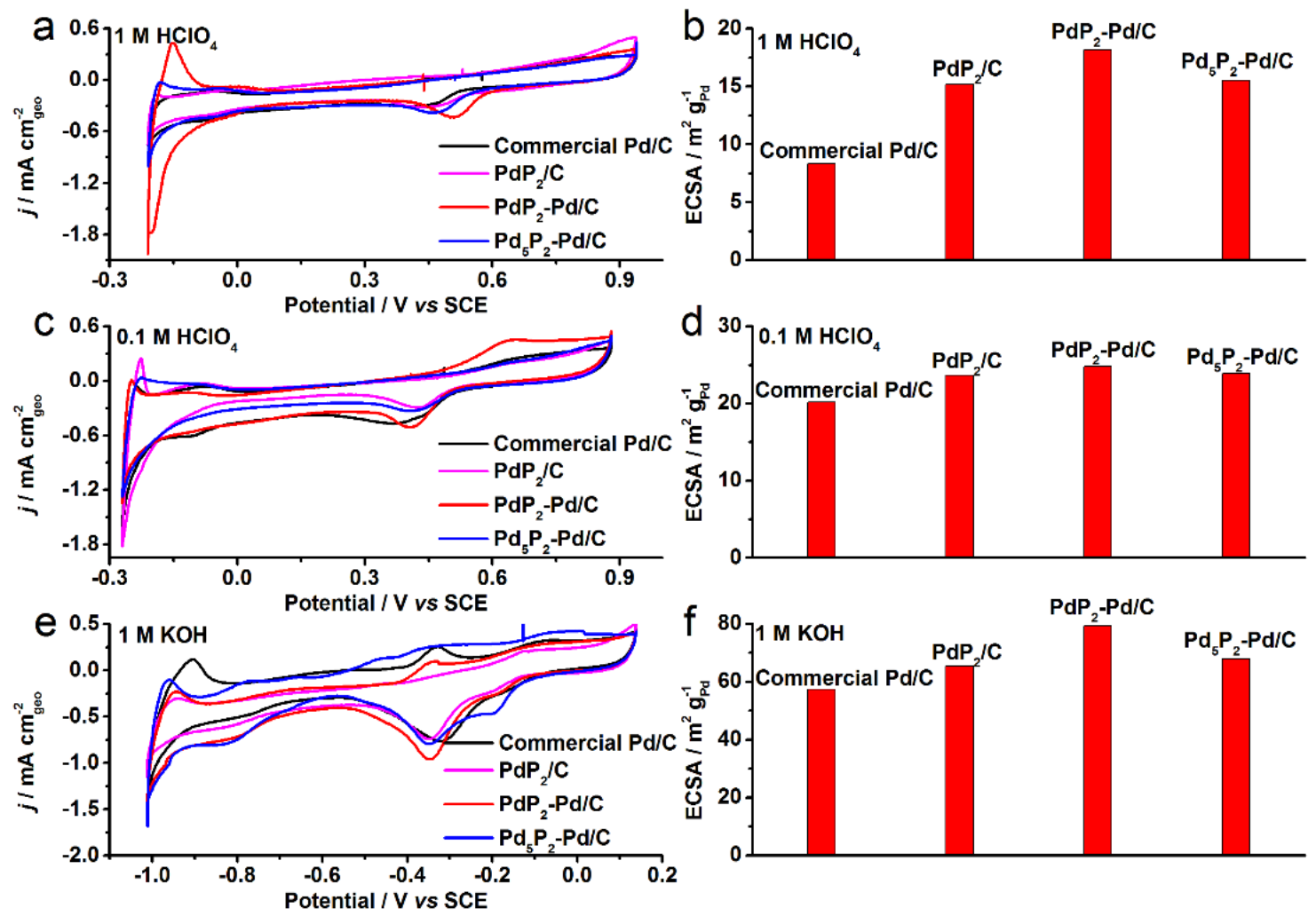

Figure S6. Electrochemical CV curves of commercial $\mathrm{Pd} / \mathrm{C}, \mathrm{PdP}_{2} / \mathrm{C}, \mathrm{PdP}_{2}-\mathrm{Pd} / \mathrm{C}$ and $\mathrm{Pd}_{5} \mathrm{P}_{2}-\mathrm{Pd} / \mathrm{C}$ catalysts recorded at a scan rates of $20 \mathrm{mV} \mathrm{s}^{-1}$ in $\mathrm{N}_{2}$-purged (a) $1.0 \mathrm{M} \mathrm{HClO}_{4}$, (c) $0.1 \mathrm{M} \mathrm{HClO}_{4}$ and (e) 1.0 M KOH. ECSAs of all Pd-based catalysts calculated from the CV curves recorded in (b) $1.0 \mathrm{M} \mathrm{HClO}_{4}$, (d) $0.1 \mathrm{M} \mathrm{HClO}_{4}$ and (f) $1.0 \mathrm{M} \mathrm{KOH}$. 

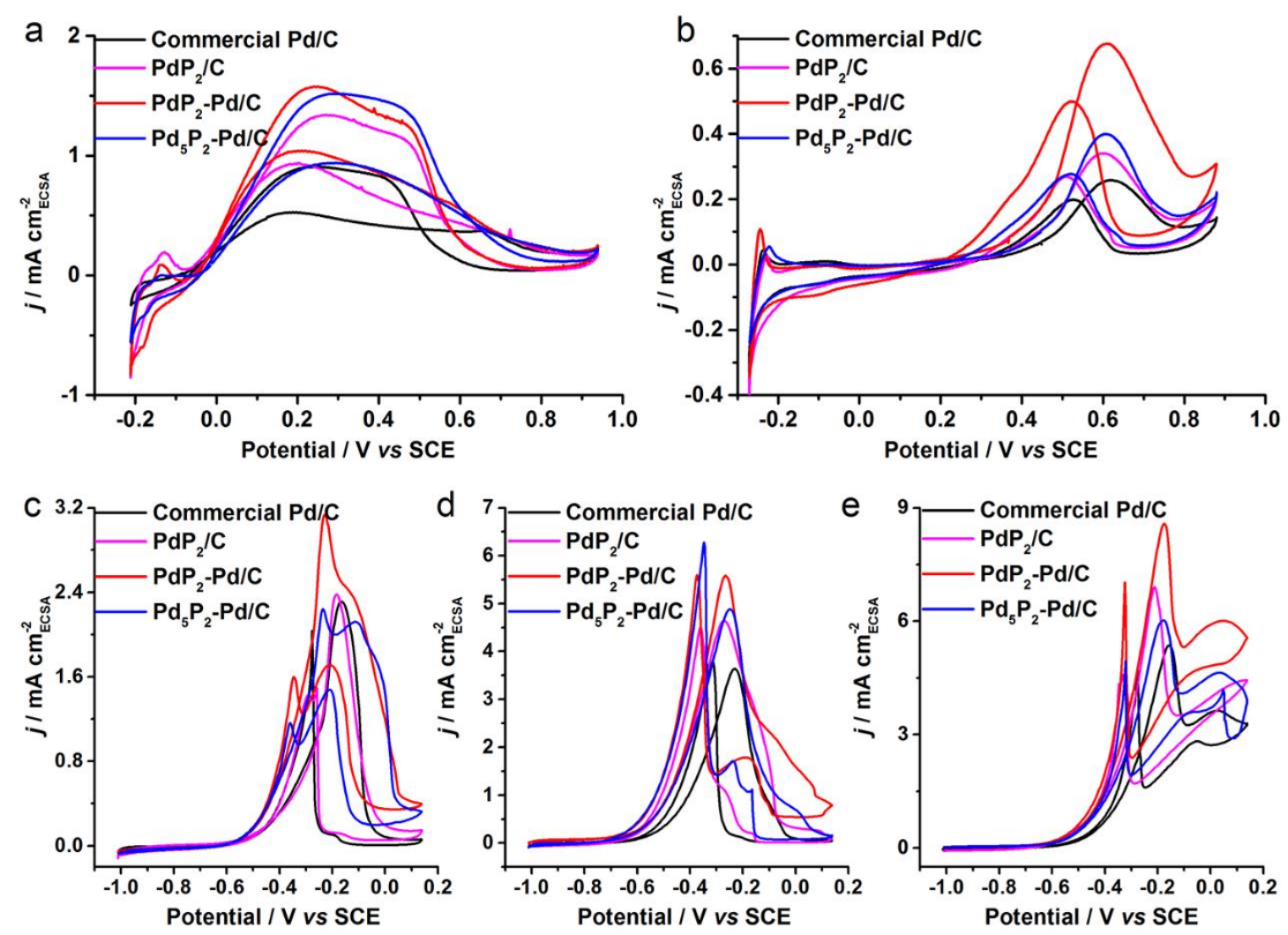

Figure S7. Specific activities of commercial $\mathrm{Pd} / \mathrm{C}, \mathrm{PdP}_{2} / \mathrm{C}, \mathrm{PdP}_{2}-\mathrm{Pd} / \mathrm{C}$ and $\mathrm{Pd}_{5} \mathrm{P}_{2}-\mathrm{Pd} / \mathrm{C}$ catalysts for different catalytic reactions. (a) FAOR-H recorded in $0.5 \mathrm{M} \mathrm{HCOOH}+1.0 \mathrm{M} \mathrm{HClO}_{4}$. (b) MOR-H recorded in $0.5 \mathrm{M} \mathrm{CH}_{3} \mathrm{OH}+0.1 \mathrm{M} \mathrm{HClO}_{4}$. (c) $\mathrm{MOR}-\mathrm{OH}$ recorded in $1.0 \mathrm{M} \mathrm{CH}_{3} \mathrm{OH}+$ 1.0 M KOH. (d) EOR-OH recorded in $1.0 \mathrm{M} \mathrm{CH}_{3} \mathrm{CH}_{2} \mathrm{OH}+1.0 \mathrm{M} \mathrm{KOH}$. (e) EGOR-OH recorded in 1.0 M EG + 1.0 M KOH. All CV curves were acquired at $20 \mathrm{mV} \mathrm{s}^{-1}$. 

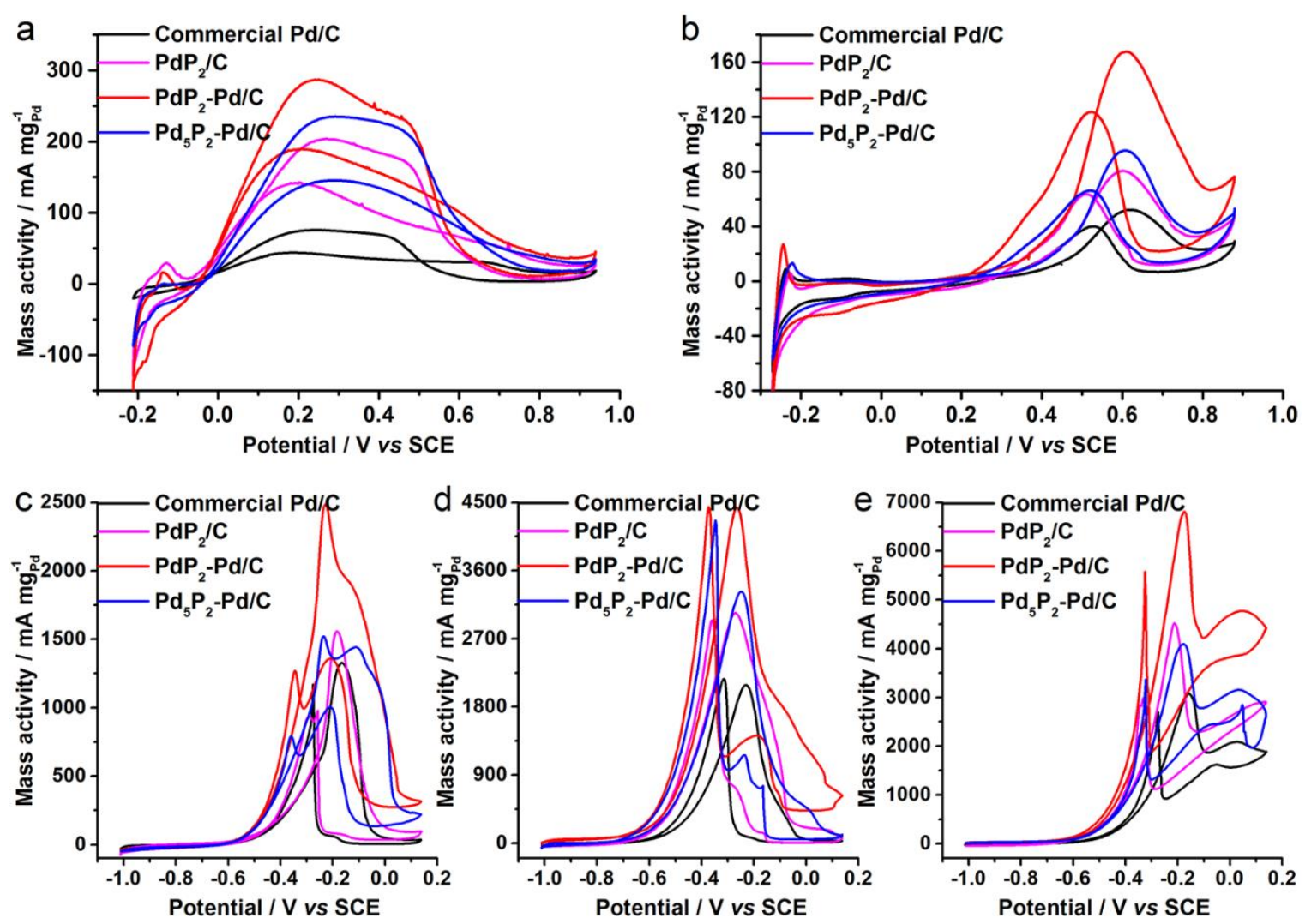

Figure S8. Mass activities of commercial $\mathrm{Pd} / \mathrm{C}, \mathrm{PdP}_{2} / \mathrm{C}, \mathrm{PdP}_{2}-\mathrm{Pd} / \mathrm{C}$ and $\mathrm{Pd}_{5} \mathrm{P}_{2}-\mathrm{Pd} / \mathrm{C}$ catalysts for different catalytic reactions. (a) FAOR-H recorded in $0.5 \mathrm{M} \mathrm{HCOOH}+1.0 \mathrm{M} \mathrm{HClO}_{4}$. (b) MOR$\mathrm{H}$ recorded in $0.5 \mathrm{M} \mathrm{CH}_{3} \mathrm{OH}+0.1 \mathrm{M} \mathrm{HClO}_{4}$. (c) $\mathrm{MOR}-\mathrm{OH}$ recorded in $1.0 \mathrm{M} \mathrm{CH}_{3} \mathrm{OH}+1.0 \mathrm{M}$ KOH. (d) EOR-OH recorded in $1.0 \mathrm{M} \mathrm{CH}_{3} \mathrm{CH}_{2} \mathrm{OH}+1.0 \mathrm{M} \mathrm{KOH}$. (e) EGOR-OH recorded in 1.0 $\mathrm{M} \mathrm{EG}+1.0 \mathrm{M} \mathrm{KOH}$. All CV curves were acquired at $20 \mathrm{mV} \mathrm{s}^{-1}$. 


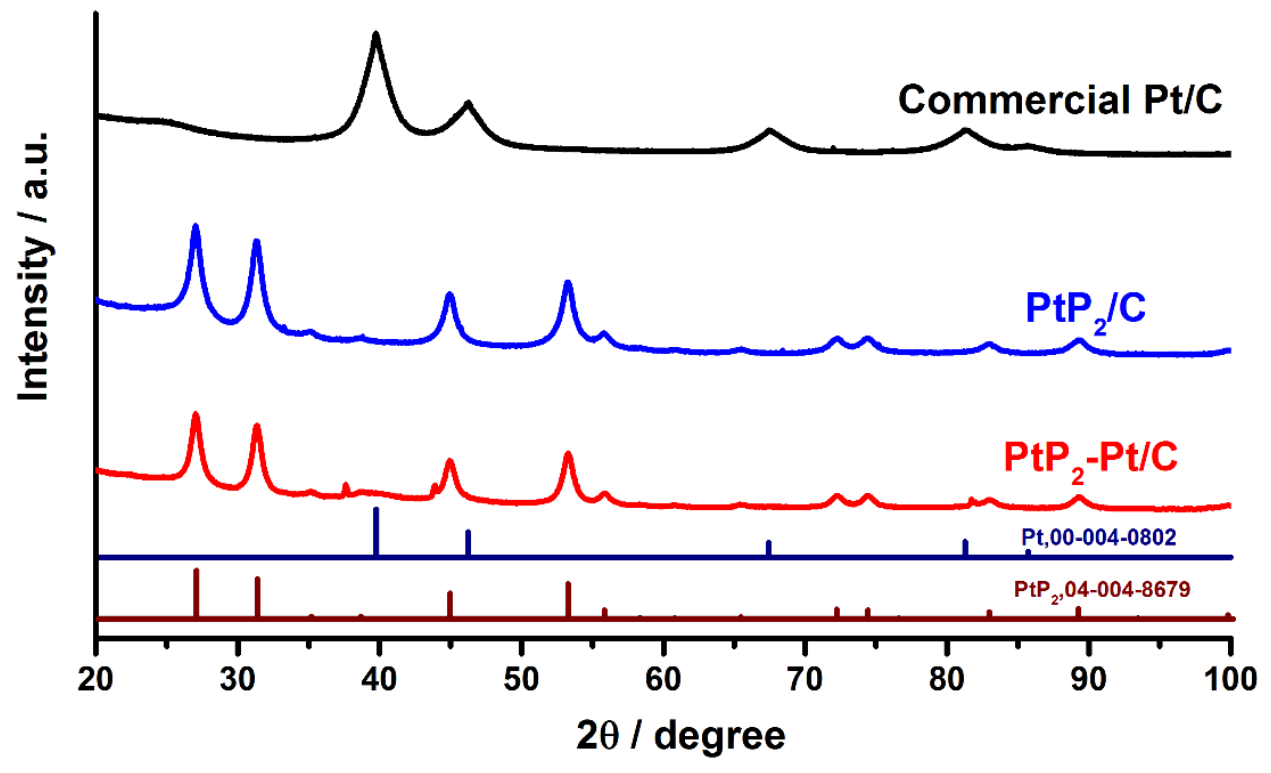

Figure S9. XRD patterns of commercial $\mathrm{Pt} / \mathrm{C}, \mathrm{PtP}_{2} / \mathrm{C}, \mathrm{PtP}_{2}-\mathrm{Pt} / \mathrm{C}$ catalysts. 

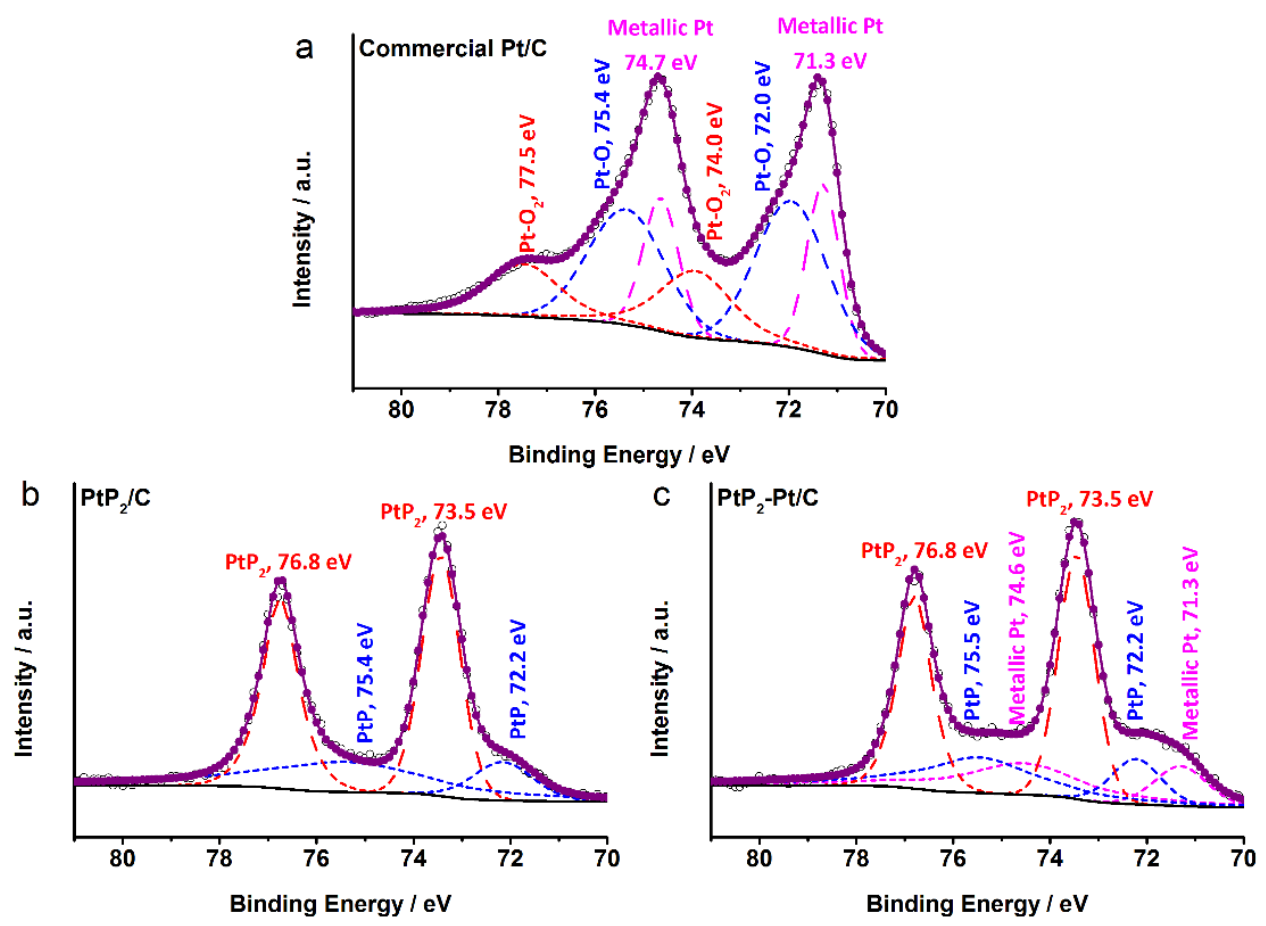

Figure S10. High resolution $\mathrm{Pt} 4 \mathrm{f}$ XPS spectra of (a) commercial $\mathrm{Pt} / \mathrm{C}$, (b) $\mathrm{PtP}_{2} / \mathrm{C}$, (c) $\mathrm{PtP}_{2}-\mathrm{Pt} / \mathrm{C}$. 

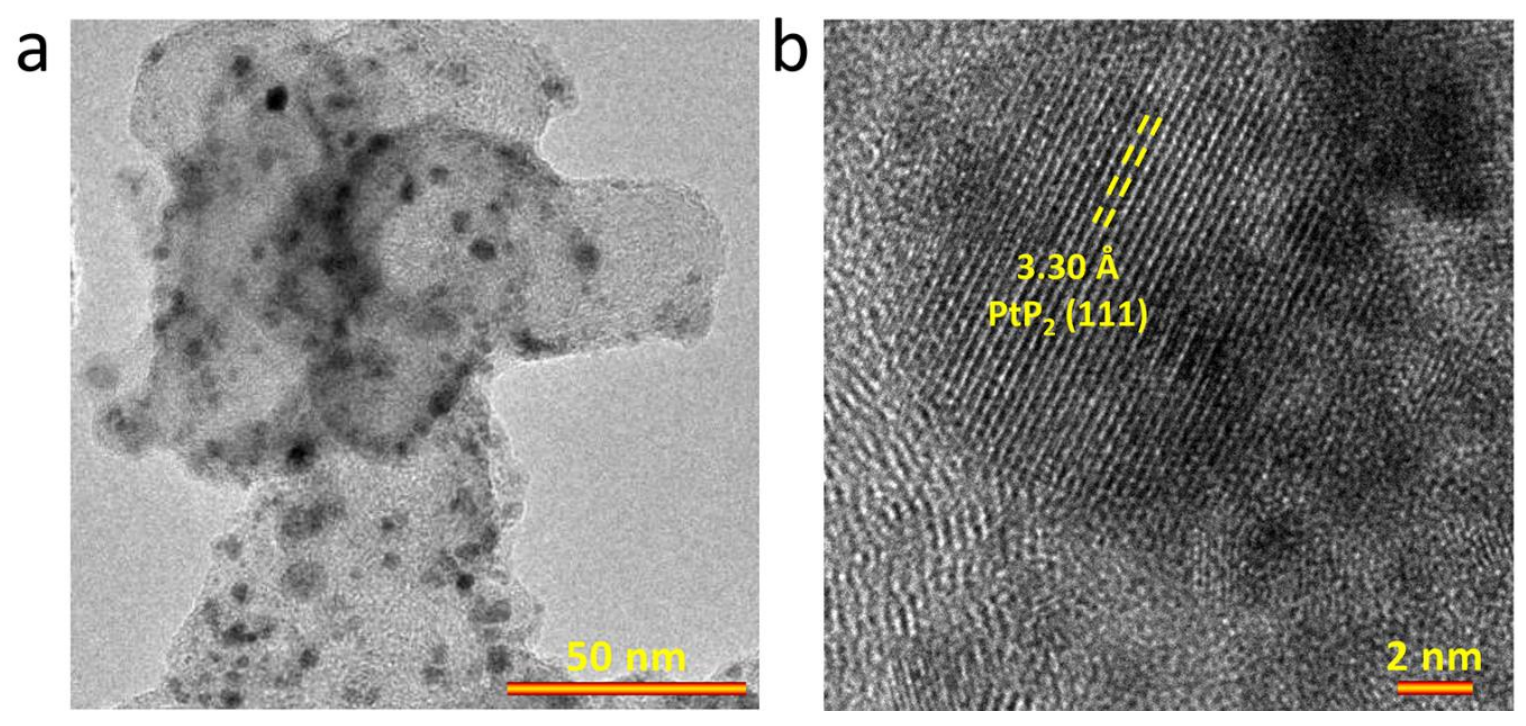

Figure S11. TEM characterization of $\mathrm{PtP}_{2} / \mathrm{C}$ catalysts. (a) Low-magnification TEM image, (b) HRTEM image. 


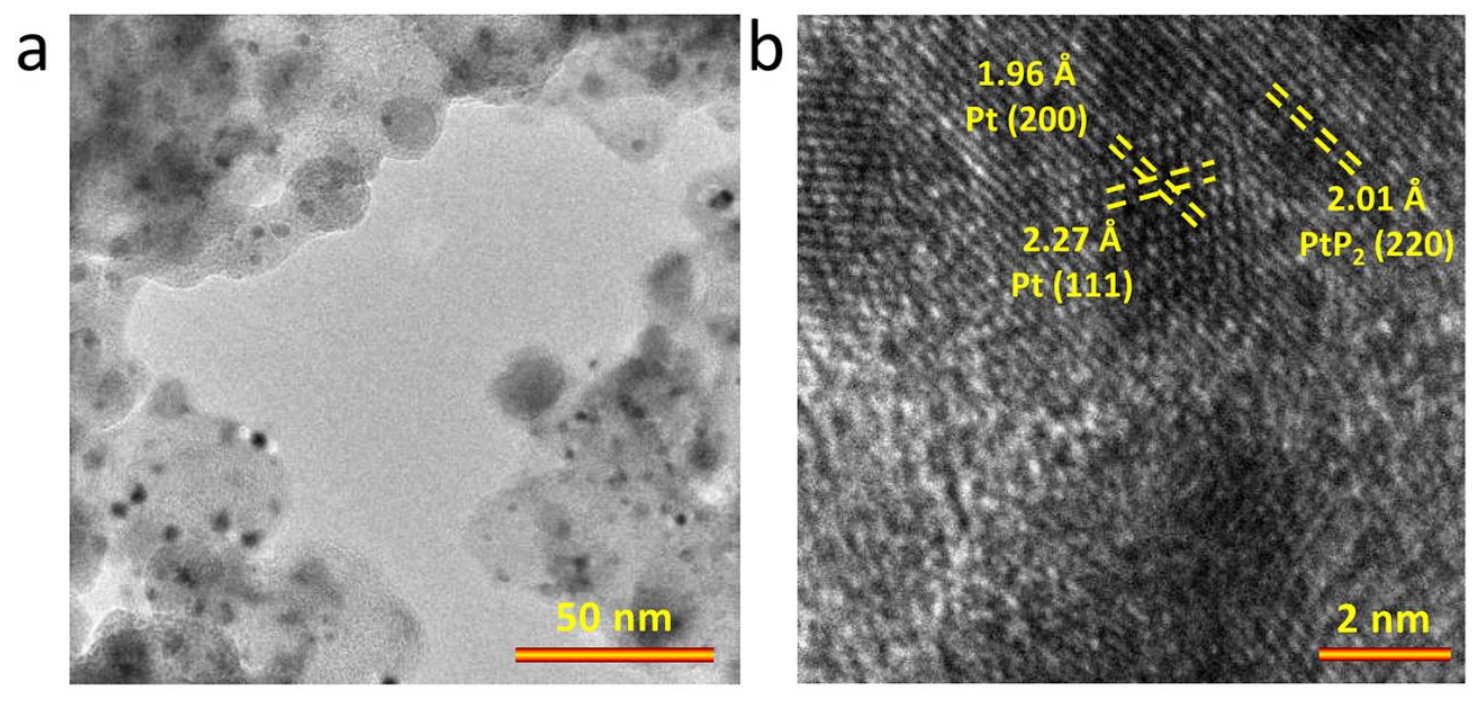

Figure S12. TEM characterization of $\mathrm{PtP}_{2}-\mathrm{Pt} / \mathrm{C}$ catalysts. (a) Low-magnification TEM image, (b) HRTEM image. 

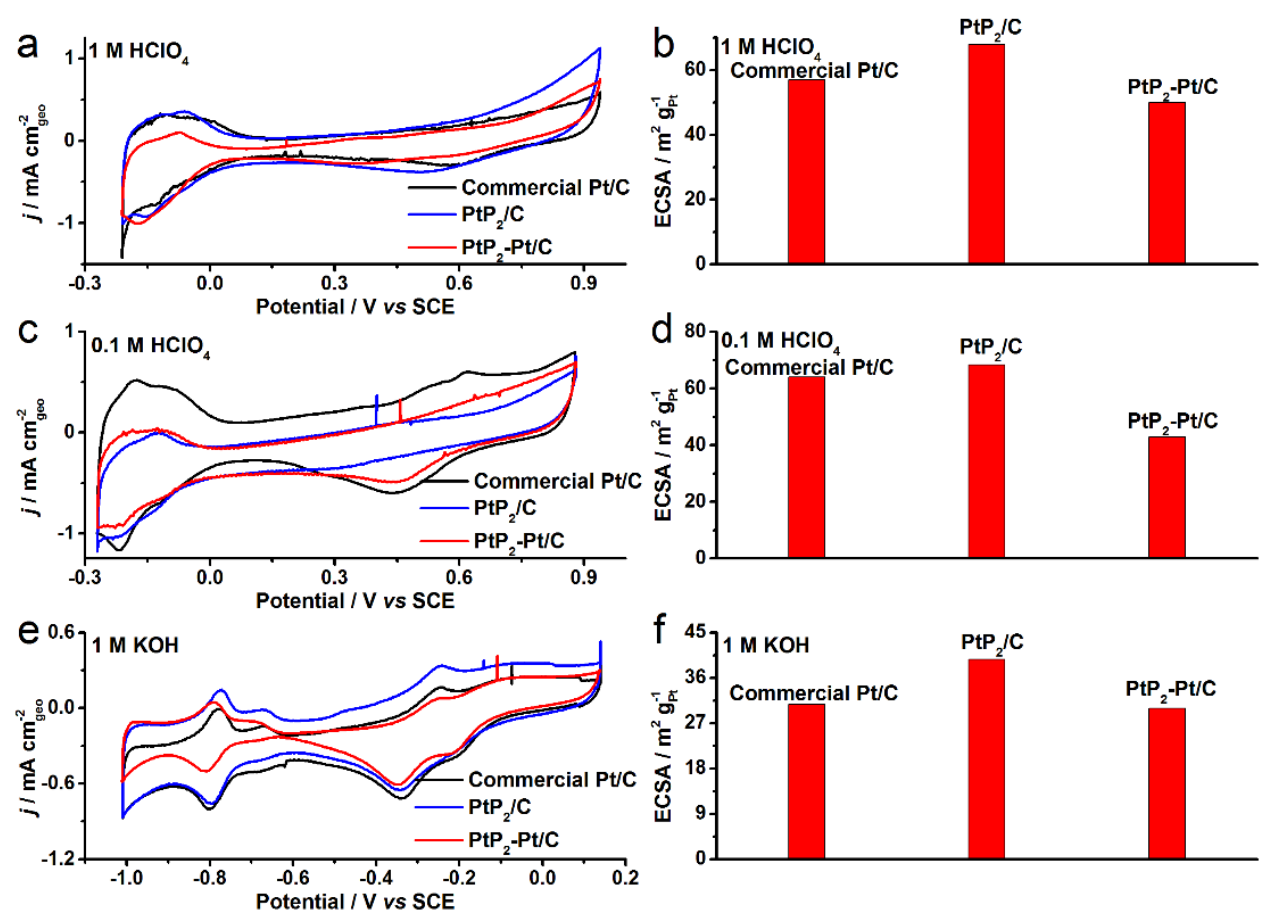

Figure S13. Electrochemical CV curves of commercial Pt/C, $\mathrm{PtP}_{2} / \mathrm{C}, \mathrm{PtP} 2-\mathrm{Pt} / \mathrm{C}$ catalysts recorded at a scan rates of $20 \mathrm{mV} \mathrm{s}^{-1}$ in $\mathrm{N}_{2}$-purged (a) $1.0 \mathrm{M} \mathrm{HClO}_{4}$, (c) $0.1 \mathrm{M} \mathrm{HClO}_{4}$ and (e) $1.0 \mathrm{M} \mathrm{KOH}$. ECSAs of all Pt-based catalysts calculated from the CV curves recorded in (b) $1.0 \mathrm{M} \mathrm{HClO}_{4}$, (d) $0.1 \mathrm{M} \mathrm{HClO}_{4}$ and (f) $1.0 \mathrm{M} \mathrm{KOH}$. 

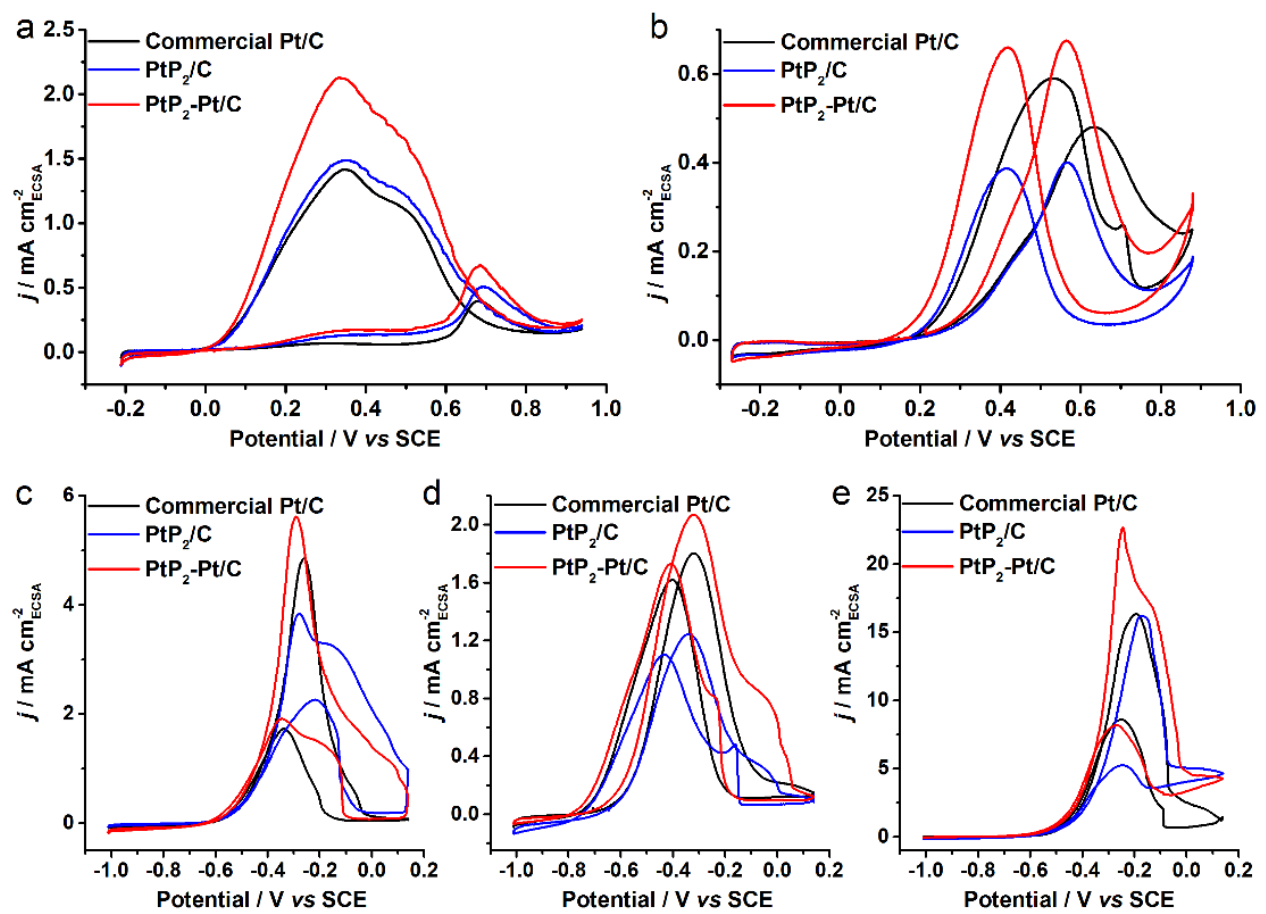

Figure S14. Specific activities and mass activities of commercial Pt/C, $\mathrm{PtP}_{2} / \mathrm{C}, \mathrm{PtP}_{2}-\mathrm{Pt} / \mathrm{C}$ catalysts for different catalytic reactions. (a) FAOR-H recorded in $0.5 \mathrm{M} \mathrm{HCOOH}+1.0 \mathrm{M} \mathrm{HClO}_{4}$. MOR-H recorded in $0.5 \mathrm{M} \mathrm{CH}_{3} \mathrm{OH}+0.1 \mathrm{M} \mathrm{HClO}_{4}$. (c) MOR-OH recorded in $1.0 \mathrm{M} \mathrm{CH}_{3} \mathrm{OH}+$ 1.0 M KOH. (d) EOR-OH recorded in $1.0 \mathrm{M} \mathrm{CH}_{3} \mathrm{CH}_{2} \mathrm{OH}+1.0 \mathrm{M} \mathrm{KOH}$. (e) EGOR-OH recorded in 1.0 M EG + 1.0 M KOH. All CV curves were acquired at $20 \mathrm{mV} \mathrm{s}^{-1}$. 

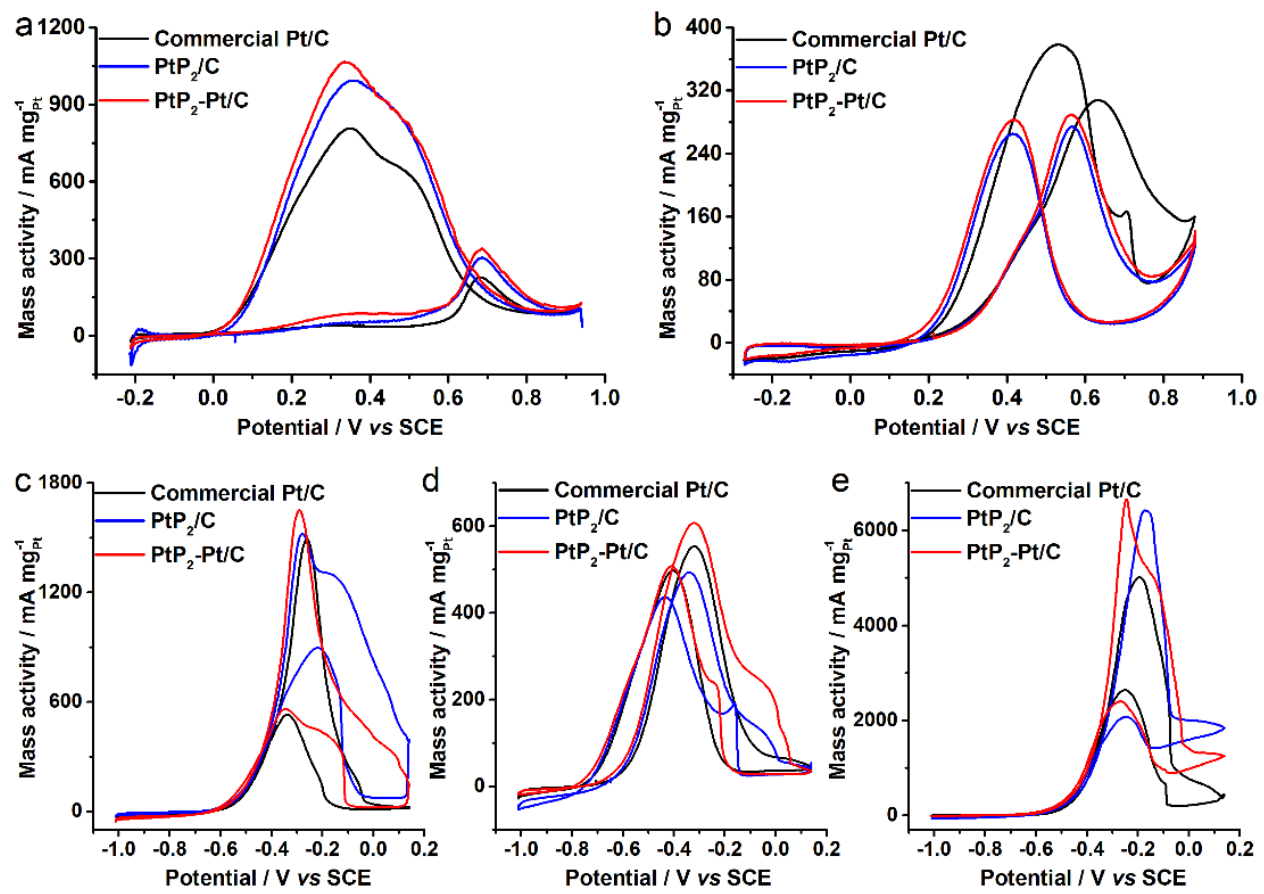

Figure S15. Mass activities of commercial Pt/C, $\mathrm{PtP}_{2} / \mathrm{C}, \mathrm{PtP}_{2}-\mathrm{Pt} / \mathrm{C}$ catalysts for different catalytic reactions. (a) FAOR-H recorded in $0.5 \mathrm{M} \mathrm{HCOOH}+1.0 \mathrm{M} \mathrm{HClO}_{4}$. (b) MOR-H recorded in 0.5 $\mathrm{M} \mathrm{CH}_{3} \mathrm{OH}+0.1 \mathrm{M} \mathrm{HClO}_{4}$. (c) MOR-OH recorded in $1.0 \mathrm{M} \mathrm{CH}_{3} \mathrm{OH}+1.0 \mathrm{M} \mathrm{KOH}$. (d) EOR-OH recorded in $1.0 \mathrm{M} \mathrm{CH}_{3} \mathrm{CH}_{2} \mathrm{OH}+1.0 \mathrm{M} \mathrm{KOH}$. (e) EGOR-OH recorded in $1.0 \mathrm{M} \mathrm{EG}+1.0 \mathrm{M} \mathrm{KOH}$. All CV curves were acquired at $20 \mathrm{mV} \mathrm{s}^{-1}$. 
Table S1. Onset potential and current densities at low potentials of different catalysts toward the OMEO reactions of investigation.

\begin{tabular}{|c|c|c|c|c|c|c|}
\hline \multicolumn{2}{|c|}{ Catalyst Reaction } & \multirow{2}{*}{$\frac{\text { FAOR-H }}{0.01}$} & \multirow{2}{*}{$\frac{\text { MOR-H }}{0.49}$} & \multirow{2}{*}{$\frac{\text { MOR-OH }}{-0.55}$} & \multirow{2}{*}{$\frac{\text { EOR-OH }}{-0.65}$} & \multirow{2}{*}{$\frac{\text { EGOH-OH }}{-0.63}$} \\
\hline \multirow{4}{*}{$\begin{array}{l}\text { Onset potential } \\
\text { (potential } \mathrm{V} \text { vs. } \\
\mathrm{SCE} \text { at } 0.5 \mathrm{~mA} \\
\mathrm{~cm}^{-2} \text { ) }\end{array}$} & $\mathrm{Pd} / \mathrm{C}$ & & & & & \\
\hline & $\mathrm{PdP}_{2} / \mathrm{C}$ & -0.01 & 0.49 & -0.49 & -0.67 & -0.65 \\
\hline & $\mathrm{PdP}_{2}-\mathrm{Pd} / \mathrm{C}$ & -0.01 & 0.42 & -0.57 & -0.80 & -0.82 \\
\hline & $\mathrm{Pd}_{5} \mathrm{P}_{2}-\mathrm{Pd} / \mathrm{C}$ & 0.01 & 0.46 & -0.56 & -0.72 & -0.82 \\
\hline \multirow{4}{*}{$\begin{array}{l}\text { Current density } \\
\text { at low potentials } \\
\left(\mathrm{mA} \mathrm{cm} \mathrm{cm}^{-2}, 0.05\right. \\
\mathrm{V} \text { for FAOR-H; } \\
0.17 \quad \mathrm{~V} \text { for } \\
\text { MOR-H; }-0.60 \\
\text { V for MOR-OH, } \\
\text { EOR-OH and } \\
\text { EGOR-OH) }\end{array}$} & $\mathrm{Pd} / \mathrm{C}$ & 0.71 & 0.02 & 0.12 & 1.19 & "0.70 \\
\hline & $\mathrm{PdP}_{2} / \mathrm{C}$ & 1.22 & 0.01 & 0.04 & 1.94 & 1.45 \\
\hline & $\mathrm{PdP}_{2}-\mathrm{Pd} / \mathrm{C}$ & 1.63 & 0.04 & 0.19 & 3.27 & 1.64 \\
\hline & $\mathrm{Pd}_{5} \mathrm{P}_{2}-\mathrm{Pd} / \mathrm{C}$ & 1.10 & 0.03 & 0.17 & 3.09 & 1.59 \\
\hline
\end{tabular}


Table S2. Comparison of the poisoning tolerance for $\mathrm{PdP}_{2}-\mathrm{Pd} / \mathrm{C}$ and $\mathrm{PtP}_{2}-\mathrm{Pt} / \mathrm{C}$ toward all reactions under investigation with respect to the commercial $\mathrm{Pd} / \mathrm{C}$ and $\mathrm{Pt} / \mathrm{C}$ catalysts.

\begin{tabular}{ccccccc}
\hline Catalysts & $\mathrm{R}$ & FAOR-H & MOR-H & MOR-OH & EOR-OH & EGOR-OH \\
\hline Commercial Pd/C & $\mathrm{I}_{\mathrm{f}} / \mathrm{I}_{\mathrm{b}}$ & 0.60 & 1.33 & 1.13 & 0.96 & 1.13 \\
$\mathrm{PdP} 2-\mathrm{Pd} / \mathrm{C}$ & $\mathrm{I}_{\mathrm{f}} / \mathrm{I}_{\mathrm{b}}$ & 0.68 & 1.40 & 1.97 & 1.00 & 1.22 \\
$\mathrm{Commercial} \mathrm{Pt/C}$ & $\mathrm{I}_{\mathrm{f}} / \mathrm{I}_{\mathrm{b}}$ & 0.28 & 0.82 & 2.81 & 1.12 & 1.90 \\
$\mathrm{PtP}_{2}-\mathrm{Pt} / \mathrm{C}$ & $\mathrm{I}_{\mathrm{f}} / \mathrm{I}_{\mathrm{b}}$ & 0.32 & 1.02 & 2.94 & 1.20 & 2.77 \\
\hline
\end{tabular}

